\title{
Research on Texture Mapping of Geological Models
}

\author{
Jixin $\operatorname{Tan}^{1,2, a}$ and Jianxun Chen ${ }^{1,2, b}$ \\ ${ }^{1}$ College of Computer Science and Technology, Wuhan University of Science and Technology, \\ Wuhan, 430065, China \\ ${ }^{2}$ Hubei province key laboratory of Intelligent information processing and Real-time Industrial System, \\ Wuhan University of Science and Technology, Wuhan, 430065, China \\ atjx1021@qq.com, bcjxwh@wust.edu.cn
}

Keywords: Texture mapping; Geological models; Parameterize; Texture coordinates; Triangular facet

Abstract. Texture mapping of 3D models is an important problem in realistic graphics. However, there are few methods that are put forward to solve texture mapping of models constructed from nature scenes for the reason that those $3 \mathrm{D}$ models are difficult to parameterize to $2 \mathrm{D}$. This paper proposes an algorithm for 3D geological models so that the texture on the side part will be well-balanced. This algorithm breaks the side part loop of 3D geological models along several edges, and unfolds the loop like a 2D plane so that the calculation of texture coordinates can be uncomplicated. At last, the algorithm is implemented and experiments are carried out to verify its effectiveness.

\section{Introduction}

Texture mapping technology is widely used in the generation and display of 3D realistic graphics $[1,3]$. Generally speaking, texture mapping has two main advantages: one is that texture mapping can describe the details of the 3D model, which enhances the realism of 3D model [4]. And another is that it simplified the modeling [5]. The essence of texture mapping is to parameterize the 3D solid model in 2-dimension. That is, first, calculate the 2-dimension parameters $(\mathrm{u}, \mathrm{v})$ of all vertices on the surface of the 3D solid model. And then, get the value of texture. Finally, generate the graphic image of the 3D model. Therefore, the parameterization is an important and complicate procedure in texture mapping. [6,7]

Geological model is a type of 3D solid model, and constructing the 3D geological model is of great importance to research the geological problems and explore and develop mineral resources [8]. Geological models are usually represented by 3D polyhedral surface and those surface mesh are constructed by nature data, which are very complicated and difficult to be parameterized.[9]

Segal proposed the method of projective mapping, [10,11]which is like project an image to a model. The calculation of texture coordinate is simple, but the texture on the models can distort easily. Paper [12] proposed two steps interactive texture mapping, which uses B-spline surface to calculate the texture coordinates, but this method needs to construct B-spline surface that approximates the 3D model and it is difficult to construct the surface from nature scenes. This paper mainly researchs the proper method to calculate the texture coordinates of vertices.

\section{Description of Problem}

A geological model is mainly divided into two parts: one is the up and down part, and another is the side part (Fig. 1-b). 


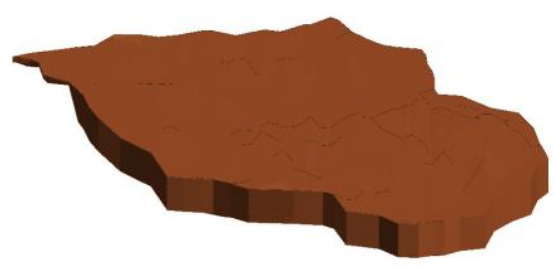

(a)

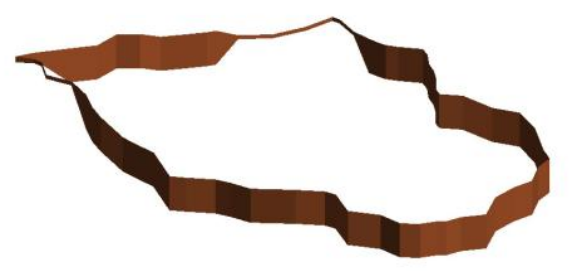

(b)

Figure 1. (a) geological model; (b) side part of geological model

Obviously, the side part is a loop and the path is irregular. As a result, the texture coordinates of vertices on the side part is difficult to calculate. In fact, the $v$ coordinate is easy to calculate.

$$
V_{i} \cdot v=V_{i} \cdot z \quad i=1,2,3, \ldots
$$

But the calculation of $u$ coordinate is difficult. The algorithm proposed in this paper is mainly deal with the calculation of $u$ coordinate. The key point of the algorithm is to break the loop and unfold it as a plane.

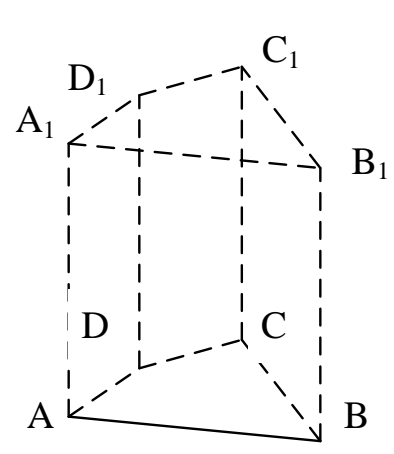

(a)

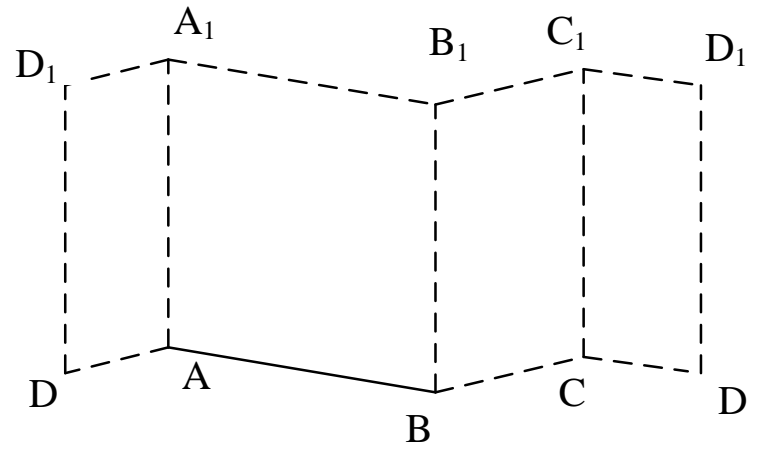

(b)

Figure 2. (a) example of geological model; (b) unfold the side part of model

This algorithm mainly has steps:

1. Pick a vertex as the base vertex whose $u$ coordinate is 0 .

2. Calculate the $u$ coordinate of an adjacent vertex of base vertex and define the positive direction.

3. Calculate the $u$ coordinate of the vertices who are adjacent to the both vertex above and define the base facet, then adjust the $u$ coordinate of vertices of the base facet.

4. Calculate other vertices based on the base facet and positive direction.

\section{Texture Mapping Algorithm}

In fact, the side part of geological models is usually presented by triangular mesh, like Fig. 3, and Fig. 3 will be taking as an example to explain this algorithm.

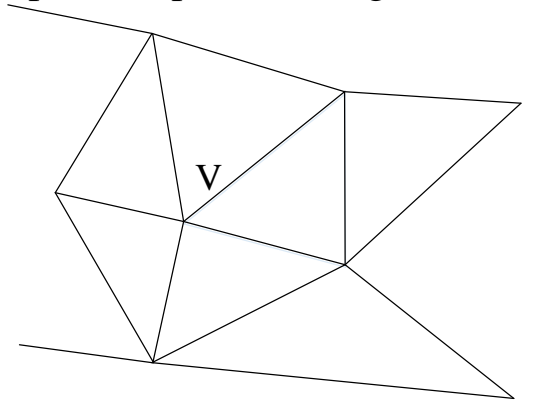

Figure 3. A side part segment of geological model 
First of all, some definitions below are necessary.

$F_{i} i=0,1,2,3, \ldots$ The sequence of the triangle facets.

$V_{i} i=0,1,2,3, \ldots$ The sequence of all vertices.

$V_{F i-j} i=0,1,2,3, \ldots j=1,2,3$ The j-th vertex of $F_{i \text { 。 }}$

$E_{V i-j} i=0,1,2,3, \ldots j=0,1,2,3, \ldots$ The edge whose two end point are $V_{i}$ and $V_{j}$.

$\vec{A}_{\text {OXY }}$ The projection of vector $\vec{A}$ on $\mathrm{z}=0$ plane.

In first step, a vertex must be chosen as the base vertex, and its $u$ coordinate is assigned 0 . In fact, any vertex can play the role. We just choose the $V_{i}$ as the base vertex. Then define the calculation direction. The calculate direction is the positive direction of this algorithm. That is to say, $V_{0}$ 's $u$ texture coordinate is 0 and the $u$ coordinate of other vertices is all positive value or 0 .

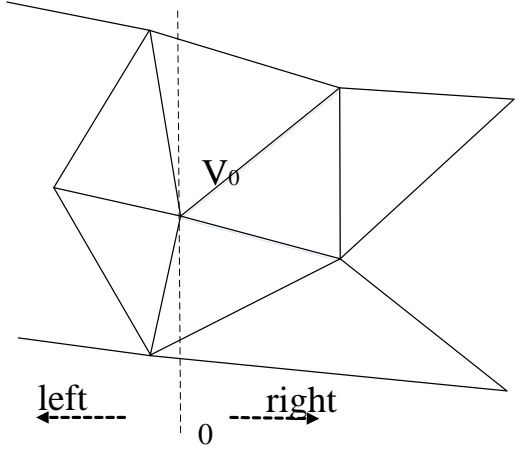

Figure 4. Define base vertex

Obviously, the direction is leftward or rightward and any direction is feasible. There are several vertices that are linked to V0. Just choose one vertex randomly, and assume the chosen vertex is V1. Then, the $\mathrm{u}$ coordinate of $\mathrm{V} 1$ can be calculated.

$$
V_{l} \cdot u=\sqrt{\left(V_{l} \cdot x-V_{0} \cdot x\right)^{2}+\left(V_{l} \cdot y-V_{0} \cdot y\right)^{2}}
$$

The positive direction of the facet is the projection of $\overrightarrow{V_{0} V_{1}}$ on $\mathrm{z}=0$ plane. Then, choose one triangle facet that has the edge EV0-1 as the base facet. Generally speaking, every edge has two facet that enclosed by it expect those who are the top edge or bottom edge of the side part. It does no matter to choose whatever facet to be the base facet. But the calculation must obey the positive direction. Assume that Fi is chosen as the base facet. Assume the V0 represented by VF0-1 and V1 represented byVF0-2 in F0. Next, calculate the u coordinate of the third vertex VF0-3.

It is necessary to judge whether the calculation is consistent with the positive direction. If the calculation keeps with the positive direction, the calculation of VF0-3's u coordinate just based VF0-1. However, if it is not, the VF0-3 will turn to be the base vertex and then calculate the $u$ coordinate of VF0-1 and VF0-2. Because the calculations will keep the positive direction.

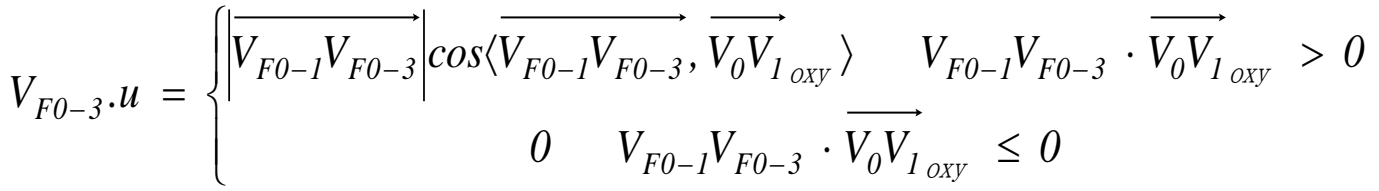

$$
\begin{aligned}
& =\left\{\begin{array}{cl}
\sqrt{\left(V_{F 0-3} \cdot x-V_{F 0-1} \cdot x\right)^{2}+\left(V_{F 0-3} \cdot y-V_{F 0-1} \cdot y\right)^{2}} & V_{F 0-1} V_{F 0-3} \cdot \overrightarrow{V_{0} V_{l_{\text {OXY }}}}>0 \\
0 & V_{F 0-1} V_{F 0-3} \cdot \overrightarrow{V_{0} V_{l_{\text {OXX }}}} \leq 0
\end{array}\right.
\end{aligned}
$$

There is one point that must be attention: that is while the condition of $V_{F O-1} V_{F O-3} \cdot \vec{V}_{0} V_{1 \text { OXY } 1}<0$ is true, VF0-1 will be defined as the base vertex. As a result, the $\mathrm{u}$ coordinates of VF0-1 (V0) and VF0-2 (V1) must be re-evaluated.

$$
V_{F O-1} \cdot u=\sqrt{\left(V_{F O-1} \cdot x-V_{F O-3} \cdot x\right)^{2}+\left(V_{F O-1} \cdot y-V_{F 0-3} \cdot y\right)^{2}}
$$




$$
V_{F O-2} \cdot u=\sqrt{\left(V_{F O-2} \cdot x-V_{F 0-3} \cdot x\right)^{2}+\left(V_{F O-2} \cdot y-V_{F O-3} \cdot y\right)^{2}}
$$

After all vertices of one facet been calculated, this facet will be signed as visited. Therefore, the $\mathrm{u}$ coordinate of other vertices can be calculated based on F0. Obviously, the projection on $\mathrm{z}=0$ plane of the vectors that constructed by the edges of the facet can determine the positive direction of the adjacent facets that share an edge with who has been visited. Here take F1 as an example.

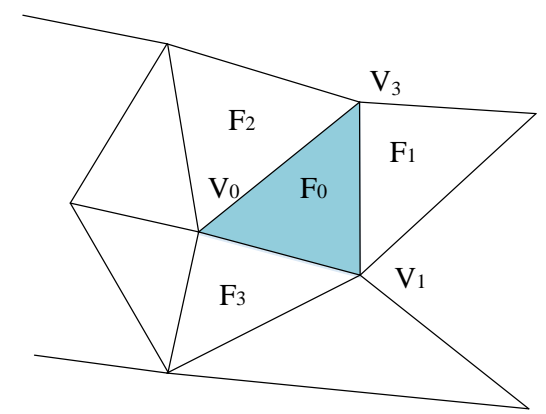

Figure 5. $F_{0}$ has been visited

In F1, VF1-1(V1) and VF1-2(V3) are also vertices of F0 and $\overrightarrow{V_{1} V_{3}}$ oxy is the positive direction of F1. The u coordinate of VF1-3 can be calculated as follows.

$$
\begin{aligned}
& \text { Factor }=\overrightarrow{V_{F 1-1} V_{F 1-3}} \cdot \overrightarrow{V_{1} V_{3}} \text { oxy } \\
& V_{F 1-3} \cdot u=\left\{\begin{array}{cc}
V_{F 1-1} \cdot u+\sqrt{\left(V_{F 1-3} \cdot x-V_{F 1-1} \cdot x\right)^{2}+\left(V_{F 1-3} \cdot y-V_{F 1-1} \cdot y\right)^{2}} & \text { Factor }>0 \\
0 \quad V_{F 1-1} V_{F 1-3} \cdot \text { Factor }=0 & \\
V_{F 1-1} \cdot u-\sqrt{\left(V_{F 1-3} \cdot x-V_{F 1-1} \cdot x\right)^{2}+\left(V_{F 1-3} \cdot y-V_{F 1-1} \cdot y\right)^{2}} & \text { Factor }>0
\end{array}\right.
\end{aligned}
$$

After been calculated, this facet will be signed visited. But be attentive, if $\mathrm{u}$ is less than 0 , this value must be discarded and this facet will not be signed visited. The remaining vertex's u coordinate will be calculated later. The whole calculation process is an iteration. After one facet been calculated, its adjacent facets will be put into a queue that records the facets to be handled.

\section{Experiment}

This experiment was operated in Windows system. The algorithm has been developed by $\mathrm{C}++$ and OpenGL. Three geological models have been used for verifying and the texture images is png format images with patterns and stripes.

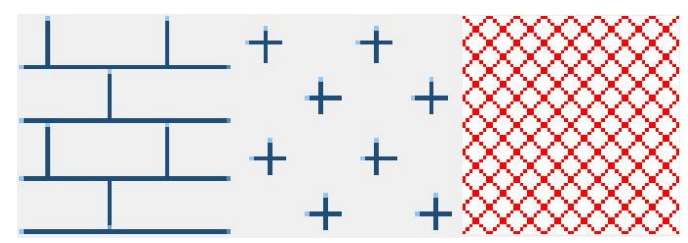

Figure 6. Texture images

The three models are the layers of one geological scene and the texture images are used to map to the side part of three layers respectively. 


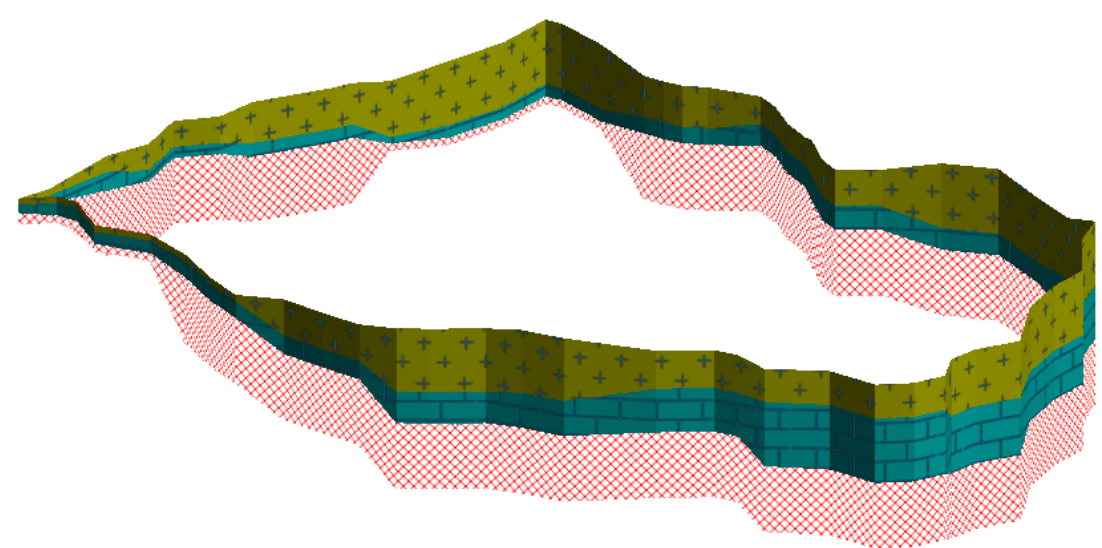

Figure 7. Map the texture to the side part of models

\section{Conclusion}

This paper focusses the problem that it is difficult to parameterize the 3D geological models so as to the texture coordinate of the models is hard to calculate and then proposed an algorithm who concentrates to texture mapping of the side part of the geological model. This algorithm defines a base vertex and the positive direction, and the texture coordinates of other vertices are all base on the base vertex. The advantages of the algorithm are that it is uncomplicated and efficient, and the performance is fine, while the weakness is that there are some distorts around base vertex.

\section{References}

[1] Peng Shengze, Guo Xingji. The Research on Texture Extraction and Mapping Implementation in 3D Building Reconstruction [J]. BULLETIN OF SCIENCE AND TECHNOLOGY, 2014,(1):72-75,81.

[2] WANG De-Cai,ZHANG Gan-Lin,PAN Xian-Zhang. Mapping Soil Texture of a Plain Area Using Fuzzy-c-Means Clustering Method Based on Land Surface Diurnal Temperature Difference[J]. Soil Science Society of China,2012,22(3):394-403.

[3] Heckbert P S. Survey of Texture Mapping[J]. IEEE Computer Graphics \& Applications, 1986, 6(11):56-67.

[4] Liu Bin, Chen Xiangning, Xue Junshi. Seamless texture mapping algorithm based on multi parameter weighted [J]. JOURNAL OF IMAGE AND GRAPHICS, 2015,20(7):929-936.DOI:10.11834/jig.20150709.

[5] FAN Bo, WU Hui-Zhong. RESEARCH ON THE TEXTURE MAPPING OF THE POLYGONAL PRIMITIVITY [J]. JOURNAL OF COMPUTER RESEARCH AND DEVELOPMENT,1999,36(4):446-450.

[6] WANG Yin, WANG Yanjie, ZHOU Yuren, LI Jingyu, YAO Zhi jun. Adaptive harmonic texture mapping algorithm based on harmonic parameter plane [J]. Chinese Journal of Liquid Crystals and Diaplays,2014,29(5):818-823.DOI:10.3788/YJYXS20142905.0818.

[7] Yao Li, Qian Shuo.REGIONAL TEXTURE MAPPING BASED ON TRIANGULAR MESH MODEL[J].Computer Applications and Software,2015,(3):205-208,223.DOI:10.3969/j.issn.1000-386x.2015.03.048.

[8] Wan Yan, Wang Huijie, Lu Jun. ON TEXTURE MAPPING BASED ON TRIANGULAR MESH MODEL[J]. Computer Applications and Software, 2016, 33(4):160-163. 
[9] JIANG Han-Qing. WANG Bo-Sheng. ZHANG Guo-Feng. BAO Hu-Jun. High-Quality Texture Mapping for Complex 3D Scenes [J].CHINESE JOURNAL OF COMPUTERS,2015,38(12):2349-2360.DOI:10.11897/SP.J.1016.2015.02349.

[10] Segal M, Korobkin C, Van Widenfelt R, et al. Fast shadows and lighting effects using texture mapping[J]. Acm Siggraph Computer Graphics, 1992, 26(2):249-252.

[11] Schmidt R, Grimm C, Wyvill B. Interactive decal compositing with discrete exponential maps[M]. ACM, 2006.

[12] TANG Jing-lei JING Xu HE Dong-jian. Study on interactive texture mapping based on two-step texture mapping[J]. COMPUTER ENGINEERING AND DESIGN,2008,29(8):2062-2064. 